\title{
De novo leaf and root transcriptome analysis to explore biosynthetic pathway of Celangulin V in Celastrus angulatus maxim
}

\author{
Weiguo Li ${ }^{1,2,3 \dagger}$, Ranran Xu ${ }^{1,2,3 \dagger}$, Xiaoguang Yan ${ }^{1,2,3}$, Dongmei Liang ${ }^{1,2,3}$, Lei Zhang ${ }^{1,2,3}$, Xiaoyu Qin 1,2,3, \\ Qinggele Caiyin ${ }^{1,2,3}$, Guangrong Zhao ${ }^{1,2,3}$, Wenhai Xiao ${ }^{1,2,3}$, Zhaonong $\mathrm{Hu}^{4,5}$ and Jianjun Qiao ${ }^{1,2,3^{*}}$
}

\begin{abstract}
Background: Celastrus angulatus Maxim is a kind of crucial and traditional insecticidal plant widely distributed in the mountains of southwest China. Celangulin $V$ is the efficient insecticidal sesquiterpenoid of $C$. angulatus and widely used in pest control in China, but the low yield and discontinuous supply impeded its further popularization and application. Fortunately, the development of synthetic biology provided an opportunity for sustainable supply of Celangulin $V$, for which understanding its biosynthetic pathway is indispensable.

Results: In this study, six cDNA libraries were prepared from leaf and root of $C$. angulatus before global transcriptome analyses using the BGISEQ-500 platform. A total of 104,950 unigenes were finally obtained with an average length of $1200 \mathrm{bp}$ in six transcriptome databases of $C$. angulatus, in which 51,817 unigenes classified into 25 KOG classifications, 39,866 unigenes categorized into $55 \mathrm{GO}$ functional groups, and 48,810 unigenes assigned to 135 KEGG pathways, 145 of which were putative biosynthetic genes of sesquiterpenoid and triterpenoid. 16 unigenes were speculated to be related to Celangulin $\vee$ biosynthesis. De novo assembled sequences were verified by Quantitative Real-Time PCR (qRT-PCR) analysis.

Conclusions: This study is the first report on transcriptome analysis of $C$. angulatus, and 16 unigenes probably involved in the biosynthesis of Celangulin $\vee$ were finally collected. The transcriptome data will make great contributions to research for this specific insecticidal plant and the further gene mining for biosynthesis of Celangulin $\vee$ and other sesquiterpene polyol esters.
\end{abstract}

Keywords: Celastrus angulatus maxim, Celangulin V, Natural insecticides, Secondary metabolism, Sesquiterpene biosynthesis, Transcriptome

\section{Background}

With the increase of global population, natural insecticides become a trend of pesticide development for their environmental friendliness. Celastrus angulatus Maxim, extensively distributed in the mountains of southwest China, have been exploited as natural insecticide resource and folk medicines attributed to their active ingredients, including sesquiterpenes ( $\beta$-agarofurans), alkaloids, and flavonoids [1].

\footnotetext{
* Correspondence: jianjunq@tju.edu.cn

${ }^{\dagger}$ Weiguo Li and Ranran Xu contributed equally to this work.

'Department of Pharmaceutical Engineering, School of Chemical Engineering and Technology, Tianjin University, Tianjin 300072, People's Republic of China ${ }^{2}$ Key Laboratory of Systems Bioengineering (Ministry of Education), Tianjin University, Tianjin 300072, People's Republic of China

Full list of author information is available at the end of the article
}

Celangulin V, the major insecticidal ingredient of C. angulatus, exhibits excellent activities against several insect species like Pieris rapae (Lepidoptera) and Locusta migratoria manilensis (Orthoptera) $[1,2]$ targeting on $\mathrm{Na}^{+} / \mathrm{K}^{+}$-ATPase (sodium/potassium pump) of these insects. As precisely identified, Celangulin V is a kind of sesquiterpene polyol esters with $\beta$-dihydroagarofuran skeleton, holding seven hydroxyl groups [1]. In the past 20 years, a series of Celangulin $\mathrm{V}$ derivates have been isolated by using bioassay-directed fractionation. A total of 44 bioactive compounds were firstly reported, displaying antifeedant, narcotic or toxic effects against some important agricultural pests $[3,4]$.

(c) The Author(s). 2019 Open Access This article is distributed under the terms of the Creative Commons Attribution 4.0 International License (http://creativecommons.org/licenses/by/4.0/), which permits unrestricted use, distribution, and reproduction in any medium, provided you give appropriate credit to the original author(s) and the source, provide a link to the Creative Commons license, and indicate if changes were made. The Creative Commons Public Domain Dedication waiver (http://creativecommons.org/publicdomain/zero/1.0/) applies to the data made available in this article, unless otherwise stated. 
Celangulin V can be extracted from roots and leaves of C. angulatus, and the content in roots is higher than that in leaves [5-7]. The current supply is dependent on filed-grown plant with unstable yield. To address such problems, attempts have been made to produce Celangulin $\mathrm{V}$ in more eco-friendly means of biosynthesis. In general, the biosynthetic pathway of sesquiterpenes could be divided into three parts. Part I is isopentenyl diphosphate (IPP) and dimethylallyl pyrophosphate (DMAPP) biosynthesis [8, 9]. Part II is carbo- cyclization of sesquiterpene biosynthesis. Part III is the modification pathway of complex functional groups in the sesquiterpene skeleton [10-13]. The whole reaction is catalyzed by a large variety of enzymes with substrate specificities (Fig. 1).

So far, the researches of $C$. angulatus mainly focused on its phytochemical properties, including the extraction of active ingredients, the identification of active substances and the evaluation of insecticidal activity [14]. As the most important bioactive component of $C$. angulatus, Celangulin V was also well studied in structures and physicochemical properties $[15,16]$. However, its biosynthetic and metabolic pathway remained poorly investigated, mainly owing to the absence of genomic or transcriptomic resources for this non-model species. Although no gene sequence information is available in public databases, we completed the transcriptome sequencing and analysis referring to the genome information of plants closely related to $C$. angulatus. Thus, comprehensive genomic information can be further analyzed for gene expression, molecular mechanisms and biological pathways $[17,18]$. This strategy has been efficiently used in traditional Chinese medicinal species such as Gardenia jasminoides [19], Pseudostellaria heterophylla [20], Panax notoginseng [21] and Cephalotaxus hainanensis [22].

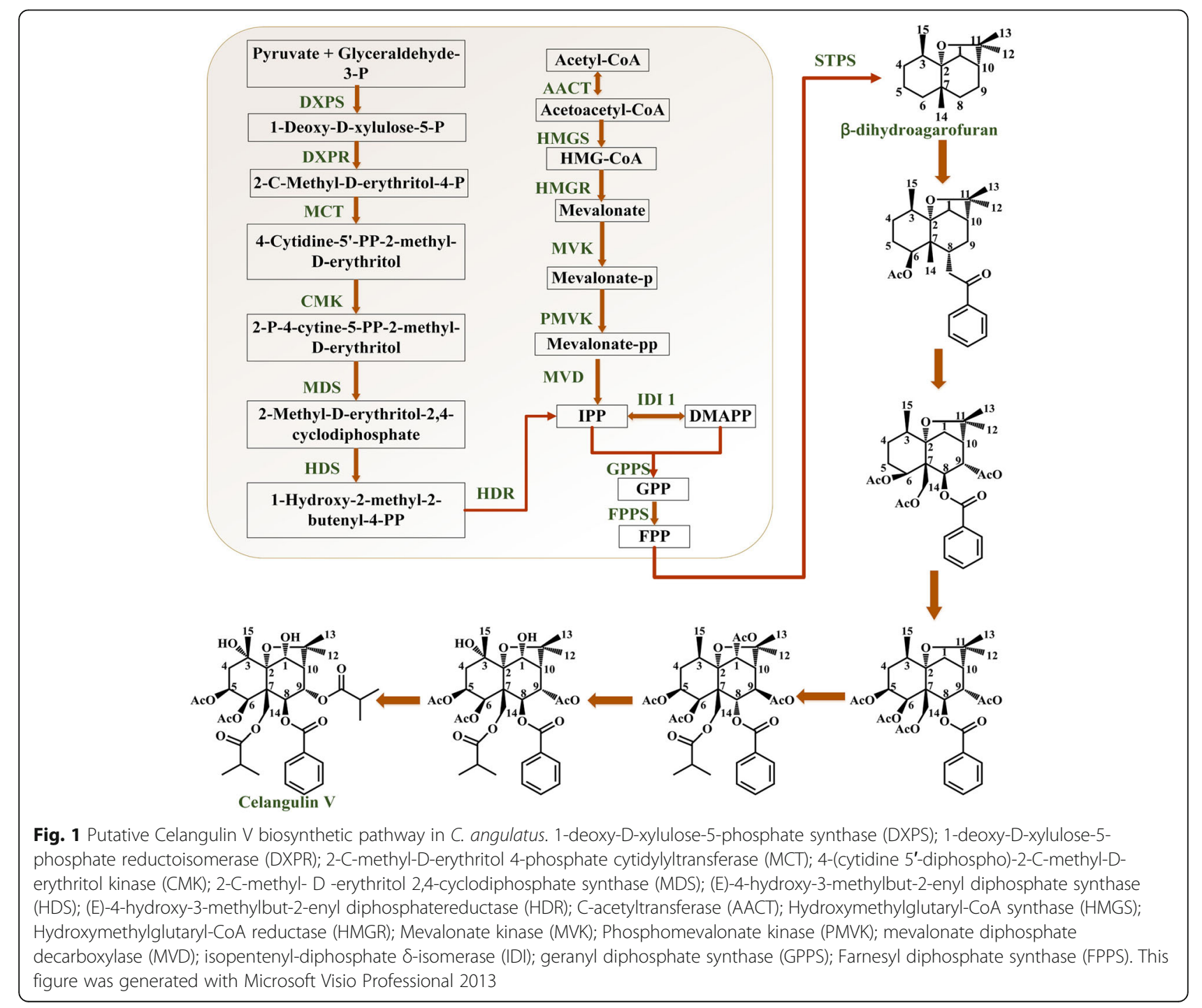


In this study, functional genes involved in sesquiterpene biosynthesis in $C$. angulatus were screened using de novo transcriptome sequencing. Four unigenes encoding sesquiterpene synthases, eight unigenes encoding cytochrome P450s (CYP450s) and four unigenes encoding acyltransferases were identified from Leaf and Root transcription databases of $C$. angulatus through BGISEQ-500. The candidate transcript sequences were listed in the Additional file 1. Differentially expressed genes (DEGs) were also analyzed. Such databases could be used as an important resource to investigate the biosynthetic pathway of Celangulin $\mathrm{V}$ in $C$. angulatus. Furthermore, this database will supply important clues to explore biological characteristics genetically in other plants with close relationship to $C$. angulatus.

\section{Methods}

\section{Plant materials}

Leaf and root tissues of $C$. angulatus were randomly picked from the exposition park of Northwest A\&F University, followed by transcriptome analysis on May 11, 2017. Leaf and root samples were harvested from three plants for RNA extraction and three biological replicates (Leaf_1 and Root_1, Leaf_2 and Root_2, Leaf_3 and Root_3) were performed. Tissues were rinsed in water, cut into small pieces, frozen in liquid nitrogen immediately, and stored at $-80{ }^{\circ} \mathrm{C}$ for further analyses. The C. angulatus was authenticated by Prof. Wenjun $\mathrm{Wu}$ and Prof. Zhaonong $\mathrm{Hu}$.

\section{cDNA library preparation and BGISEQ-500 sequencing for transcriptome analysis}

Total RNA was extracted using an RNA plant Plus Reagent (Tiangen, Beijing, China) according to the manufacturer's protocol. The extracted RNA was checked using a NanoDrop 2000 (Thermo, CA, USA), and the RNA concentration and integrity were assessed using the RNA Nano 6000 Assay Kit of the Agilent Bioanalyzer 2100 system (Agilent, CA, USA) to ensure that the RNA Integrity Number (RIN) values were above 7.0.

Oligo (dT) beads were used to isolate poly $(\mathrm{A})+$ mRNA, which was fragmented to $250 \mathrm{bp}$. Fragmentation of the RNA and reverse transcription of double-strand cDNA (ds cDNA) by N6 random primer. The synthesized cDNA was subjected to end-repair and then was 3 ' adenylated. Adaptors were ligated to the ends of these 3' adenylated cDNA fragments; The ligation products were purified and many rounds of PCR amplification were performed to enrich the purified CDNA template using PCR primer; Denature the PCR product by heat and the single strand DNA is cyclized by splint oligo and DNA ligase; Each cDNA library was sequenced in a single lane of the BGISEQ-500 system with paired-end sequencing length of $100 \mathrm{bp}$ according to the manufacturer's instructions at the Beijing Genomics Institute (BGI-Shenzhen, China). The amounts of reads generated per sample was $10-11 \mathrm{~Gb}$ to obtain deep coverage of transcripts for de novo assembly.

\section{De novo assembly and functional annotation analysis of BGISEQ-500 sequencing}

To obtain high-quality clean read data for de novo assembly, the raw reads from BGISEQ-500 were filtered by discarding the reads with adaptor sequences, reads with ambiguous "N" bases larger than 5\% and removing the low-quality reads in which more than $20 \%$ bases had a $\mathrm{Q}$-value $<15$. All the downstream analyses were based on the resulting clean reads. We used the software Trinity (v2.0.6) [23], which was efficient to form contigs in reconstructing full-length transcripts across a broad range of expression levels and sequencing depths, with default parameters and a minimum contig length of 150 bp for assembly generation. These contigs were then further processed with sequence clustering software TGICL (v2.0.6) [24] to remove the redundant Trinity generated contigs.

To determine the functional annotation of the unigenes, a BLASTx search was performed with an E-value of $10^{-5}$ against protein databases, including $\mathrm{Nr}$ (non-redundant) protein database, SwissPort, KOG (euKaryotic Orthologous Group database), and KEGG (Kyoto Encyclopedia of Genes and Genomes protein database). Besides, a BLASTn search was also performed against the Nt database. With $\mathrm{Nr}$ annotation, the Blast2GO [25] and InterProScan5 program was used to obtain the GO (Gene ontology) and InterPro annotation of unigenes, respectively. GO classification was then performed using WEGO software [26] to illustrate the distribution of gene functions including Biological Process, Cellular Component and Molecular Function.

\section{Differentially expressed unigene analysis}

After assembly, clean reads were mapped to unigenes using Bowtie2 (v2.2.5) [27], and then gene expression level was calculated with RSEM (v1.1.12) [28]. To compare the difference of gene expression among different samples, the FPKM (Fragments per kilobase per transcript per million mapped reads) method was used [29]. The DEseq2 (Fold Change $>=2.00$ and Adjusted Pvalue $<=0.05$ ) and PossionDis (Fold Change $>=2.00$ and $\mathrm{FDR}<=0.001$ ) were proposed to identify DEGs, and the $P$-value and FDR (false discovery rate) for each gene were calculated. DEGs were required to have thresholds of " $\log 2$ ratio $\geq 1$ " and "FDR < 0.001" [30]. Next, GO and KEGG analysis were again performed on the DEGs. 


\section{Phylogenetic analysis}

The phylogeny was inferred using the Neighbor-Joining method [31]. The bootstrap consensus tree inferred from 500 replicates is taken to represent the evolutionary relationship of the taxa analyzed [32]. Branches corresponding to partitions reproduced in less than $50 \%$ bootstrap replicates are collapsed. The percentage of replicate trees in which the associated taxa clustered together in the bootstrap test (500 replicates) are shown next to the branches. The analysis involved 38 terpene synthase and 52 CYP450 nucleotide sequences, respectively. All positions containing gaps and missing data were eliminated. There was a total of 479 and 1094 positions in the final dataset, respectively. Evolutionary analyses were conducted in MEGA7 [33].

\section{qRT-PCR analysis}

Total RNA was extracted as indicated above. Each RNA sample was treated with RNase-free DNase (Tiangen, Beijing, China) following the manufacturer's protocol in an effort to remove any residual genomic DNA (gDNA). DNase-treated RNA (2 mg) was subjected to reverse transcriptase reactions using M-MLV reverse transcriptase (Tiangen, Beijing, China) according to the manufacturer's instructions. The sequences of the specific primer sets are listed in Additional file 2. The Actin (CL5382.Contig2_All) gene was used as an endogenous control, qRT-PCR was performed in 96-well plates in a Bio-Rad CFX96 real-time PCR system (Bio-Rad, CA, USA) with a SYBR Green-based PCR assay. The final volume for each reaction was $20 \mu \mathrm{L}$ with the following components: $2 \mu \mathrm{L}$ diluted cDNA template $(1 \mathrm{mg} / \mathrm{mL})$, $10 \mu \mathrm{L}$ SYBR Green Mix (Bio-Rad, CA, USA), $0.4 \mu \mathrm{L}$ forward primer $(10 \mu \mathrm{M}), 0.4 \mu \mathrm{L}$ reverse primer $(10 \mu \mathrm{M})$ and $7.2 \mu \mathrm{L} d \mathrm{ddH}_{2} \mathrm{O}$. The reaction was conducted under the following conditions: $95^{\circ} \mathrm{C}$ for $3 \mathrm{~min}$, followed by $40 \mathrm{cy}$ cles of denaturation at $95^{\circ} \mathrm{C}$ for $10 \mathrm{~s}$ and $60^{\circ} \mathrm{C}$ for $30 \mathrm{~s}$. The melting curve was obtained by heating the amplicon from $65^{\circ} \mathrm{C}$ to $95^{\circ} \mathrm{C}$ at increments of $0.5^{\circ} \mathrm{C}$ per $5 \mathrm{~s}$. Each qRT-PCR analysis was performed with three biological replicates. The relative quantification of gene expression was computed using the $2^{-\Delta \Delta \mathrm{Ct}}$ method.

\section{Results}

\section{Active ingredient content in different tissues of $C$.} angulatus

Prof. Wenjun Wu have detected the active ingredient content in different tissues of $C$. angulatus. He extracted the adhesive paste of the root bark, bark, xylem, leaves and fruits of $C$. angulatus and determined the bioactivities of the extracts by feed poisoning method. As a result, the active ingredients of $C$. angulatus were mainly distributed in the root bark, secondly in the leaves. However, bark and xylem contained little active ingredients, and the extracts from the fruit stimulated the growth of larvae of Mythimna separate at low concentrations (Table 1) [34] .

\section{C. angulatus transcriptome sequencing and unigene assembly}

In order to explore gene expression profile of $C$. angulatus, six cDNA libraries prepared from three leaf tissues (Leaf_1, Leaf_2 and Leaf_3) and three root tissues (Root_1, Root_2 and Root_3) were sequenced using the BGISEQ-500 platform. A total of 110.16, 109.15, 109.87, $109.56,110.68$, and $109.52 \mathrm{Mb}$ clean reads were obtained after eliminated the low-quality reads (Table 2). Subsequently, the assembly of those clean reads were carried out and 77,255, 67,225, 69,274, 60,493, 56,701 and 60,373 unigenes were acquired by Trinity with a mean length of $970,961,961,927,839$ and 949 bp respectively (Table 3).

After de novo assembly of two $C$. angulatus tissues, 104,950 unigenes were finally obtained with an average length of $1200 \mathrm{bp}$ (Table 3). Among these, 48,715 unigenes have a length longer than $1 \mathrm{~kb}(>1000 \mathrm{bp})$ and 16,934 unigenes have a length range between $1000 \mathrm{bp}$ and $1500 \mathrm{bp}$. A detailed summary of the sequencing and assembly results is shown in Table 3 and the length distribution of all unigenes is shown in Fig. 2.

\section{Functional annotation of $C$. angulatus unigenes}

The contigs in six transcriptome sequencing databases were integrated and assembled into a total of 104,950 unigenes. After the assembly, annotations for the assembled unigenes were carried out via BLAST in seven public databases (NR, NT, Swissprot, KEGG, KOG, InterPro and $\mathrm{GO}$ ), a total of $66,193,53,315,44,141,48,810,51,817$, 56,377 and 39,866 were aligned, respectively. There were 71,479 unigenes (68.11\% of all unigenes) annotated within at least one functional database. (Additional file 3).

\section{Functional classification of $C$. angulatus unigenes by KOG, GO and KEGG}

KOG classification was used to further evaluate the completeness and effectiveness of the C. angulatus annotation.

Table 1 Effects of extracts from different tissues of $C$. angulatus on larvae of M. separate [34]

\begin{tabular}{lllll}
\hline Tissues & $\begin{array}{l}\text { Extraction } \\
\text { rate } / \%\end{array}$ & $\begin{array}{l}\text { Concentration } \\
\text { in feed } /(\mathrm{mg} / \mathrm{kg})\end{array}$ & $\begin{array}{l}\text { Weight of } \\
\text { larvae } 7 \text { days } \\
\text { later } / \mathrm{mg}\end{array}$ & $\begin{array}{l}\text { Growth } \\
\text { inhibition } \\
\text { rate } / \%\end{array}$ \\
\hline Root bark & 7.5 & 200 & 2.2 & 91.6 \\
Leave & 8.4 & 200 & 12.1 & 53.3 \\
Xylem & 1.6 & 200 & 18.1 & 30.0 \\
Bark & 3.7 & 200 & 19.5 & 24.6 \\
Fruit & 16 & 200 & 31.4 & -21.5 \\
\hline
\end{tabular}


Table 2 Summary of data output quality of various libraries

\begin{tabular}{cllll}
\hline Sample & Total Raw Reads (Mb) & Total Clean Reads (Mb) & Q20\% & Q30\% \\
\hline Leaf_1 & 135.45 & 110.16 & 95.78 & 87.48 \\
Leaf_2 & 129.99 & 109.15 & 96.09 & 88.03 \\
Leaf_3 & 132.88 & 109.87 & 95.98 & 87.93 \\
Root_1 & 135.37 & 109.56 & 95.94 & 87.91 \\
Root_2 & 135.41 & 110.68 & 95.97 & 88.10 \\
Root_3 & 129.81 & 109.52 & 96.33 & 88.83
\end{tabular}

Q20: The percentage of bases with a Phred value $>20$

Q30: The percentage of bases with a Phred value $>30$

51,817 unigenes were classified into 25 functional classifications (Fig. 3). The most dominant term was "General function prediction only" and 12,607 unigenes (24.3\%) matched it, followed by "Signal transduction mechanisms" (6864, 13.3\%), "Posttranslational modification, protein turnover, chaperones" and so on. Notably, 1768 unigenes in the "secondary metabolites biosynthesis transport and catabolism" category may play important roles in the biosynthesis of Celangulin V and other secondary metablites.

$\mathrm{GO}$ analysis is an international standard system of gene function classification, and the mainly terms including "biological process", "cellular component" and "molecular function". Among the 104,950 unigenes, 39,866 unigenes were assigned to 55 GO terms. 24 groups were involved in biological process, in which "metabolic process" and "cellular process" occupied the mainly categories. 17 groups were mainly existed in "cellular component" and the high percentage of unigenes was associated with "cell", "cell part" and "membrane". 14 groups existing in "molecular

Table 3 Summary of assembly results of $C$. angulatus

\begin{tabular}{llllll}
\hline Sample & $\begin{array}{l}\text { Total } \\
\text { number }\end{array}$ & Total length & $\begin{array}{l}\text { Mean } \\
\text { length }\end{array}$ & N50 & GC(\%) \\
\hline Leaf_1-Conting & 109,851 & $94,892,188$ & 863 & 1648 & 41.71 \\
Leaf_2-Conting & 95,389 & $82,261,657$ & 862 & 1593 & 41.82 \\
Leaf_3-Conting & 98,035 & $84,748,426$ & 864 & 1609 & 41.67 \\
Root_1-Conting & 86,993 & $72,550,947$ & 833 & 1538 & 42.16 \\
Root_2-Conting & 83,316 & $66,867,324$ & 802 & 1460 & 42.41 \\
Root_3-Conting & 86,799 & $73,974,071$ & 852 & 1589 & 42.27 \\
Leaf_1-Unigene & 77,255 & $74,938,903$ & 970 & 1720 & 41.65 \\
Leaf_2-Unigene & 67,225 & $64,632,431$ & 961 & 1657 & 41.76 \\
Leaf_3-Unigene & 69,274 & $66,633,515$ & 961 & 1659 & 41.62 \\
Root_1-Unigene & 60,493 & $56,082,948$ & 927 & 1583 & 42.11 \\
Root_2-Unigene & 56,701 & $50,673,238$ & 893 & 1504 & 42.38 \\
Root_3-Unigene & 60,373 & $57,304,625$ & 949 & 1637 & 42.21 \\
All- Unigene & 104,950 & $125,979,466$ & 1200 & 2011 & 41.61 \\
\hline
\end{tabular}

N50: a weighted median statistic that $50 \%$ of the Total Length is contained in Transcripts great than or equal to this value function", "catalytic activity" and "binding functions" play the most assignments in this category (Fig. 4).

A total of 48,810 unigenes were assigned to 135 KEGG pathways. The "metabolic pathways" represented the greatest group (9865 unigenes, 20.21\%), followed by "biosynthesis of secondary metabolites" (5011 unigenes, 10.27\%), including sesquiterpenoid and triterpenoid biosynthesis, terpenoid backbone biosynthesis, phenylpropanoid biosynthesis, cyanoamino acid metabolism, carotenoid biosynthesis and so on (Table 4). These pathways provide a valuable reference for investigating specific processes, functions and pathways during $C$. angulatus development.

The sesquiterpene polyol ester Celangulin $\mathrm{V}$ is the major insecticidal active component in C. angulatus. 145 unigenes involved in sesquiterpenoid and triterpenoid biosynthesis were found in the KEGG pathways, and the detailed metabolic pathway is shown in Additional file 4 . The pathway will be useful for further studies on the biosynthesis of Celangulin V.

\section{DEGs in the leaf vs root in C. angulatus}

DEGs of the six transcriptome libraries were used to discover the unigenes with significant differences in expression. The expression of unigenes was calculated by FPKM. The different analysis methods were as follows: Leaf_1 Vs Root_1, Leaf_2 Vs Root_2, and Leaf_3 Vs Root_3 libraries were analyzed, and the DEGs were used to comment on all three replicates for GO classification and KEGG pathway analysis. A total of 19,440 DEGs were obtained, including 9024 up-regulated and 10,416 down-regulated unigenes in Leaf Vs Root. Furthermore, 5667, 1994 and 2120 unigenes expressed uniquely in Leaf_1, Leaf_2, and Leaf_3, respectively, and 75,738 unigenes were expressed in all three libraries, but at different levels; 3612, 2621 and 4541 unigenes expressed uniquely in Root_1, Root_2 and Root_3, respectively, and 70,006 unigenes were expressed in all three libraries, but at different levels (Fig. 5).

A total of 11,948 DEGs could be annotated in KEGG based on sequence homologies, which were annotated into 135 pathways and most genes were related to metabolic pathways (2854 DEGs) and biosynthesis of secondary metabolites (1764 DEGs). For the GO classification analysis, about 63667082 and 7890 unigenes could be annotated in "biological process", "cellular component", "molecular function" of GO terms respectively. Furthermore, the number of up-regulated genes was more than the number of down-regulated genes in the three pathways.

Biosynthetic genes of terpenoid backbone in $C$. angulatus Based on the KEGG annotation, a total of 310 contigs/unigenes are correlated with the terpene 


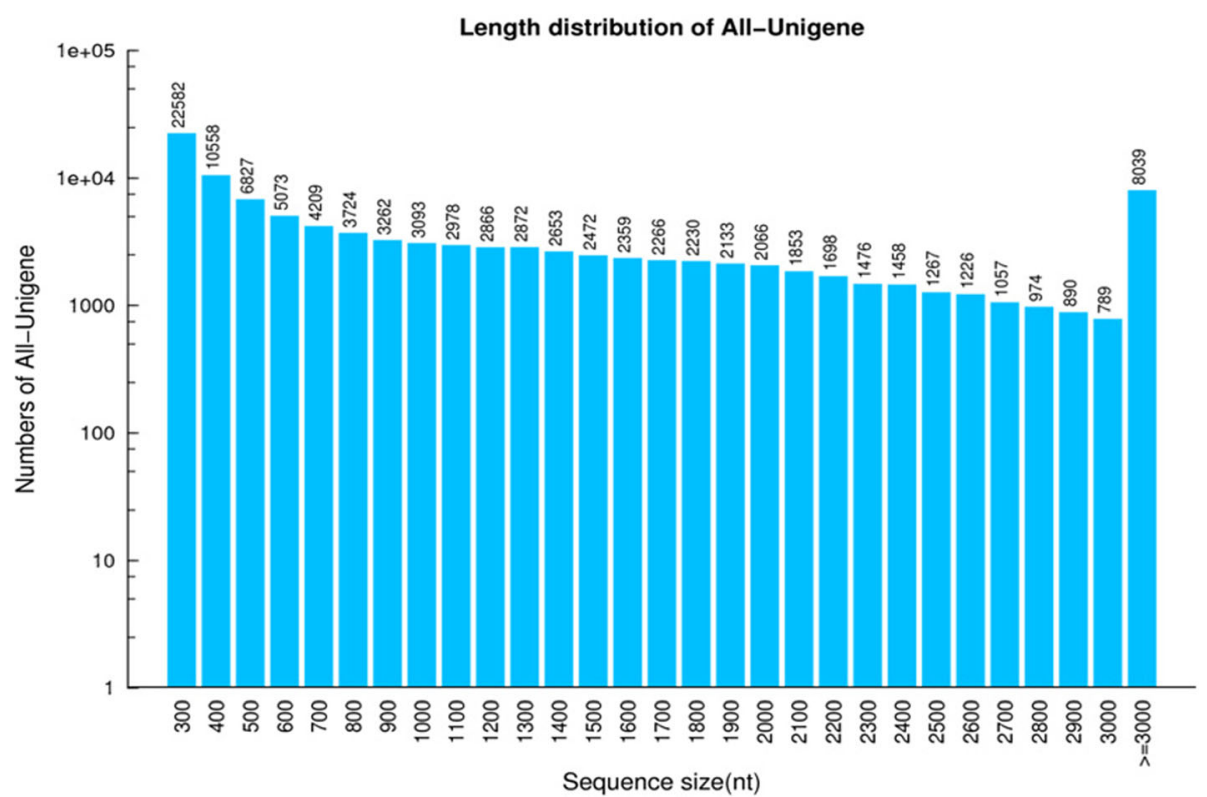

Fig. 2 Distribution size of de novo assembled unigenes for C. angulatus. A total of 104,950 unigenes sizes were calculated for C. angulatus. This figure was generated with R 3.2.5

backbone biosynthesis, accounting for $0.64 \%$ among all genes with pathway annotation $(48,810)$. Among them, 119 DEGs were discovered, accounting for $1 \%$ DGEs with pathway annotation $(11,948)$. The unigenes encoding key enzymes involved in terpenoid backbone biosynthesis were listed in Table 5. 22 up-regulated unigenes among 26 DEGs were related to Mevalonate (MVA) pathway, including seven contigs/unigenes for acetyl-CoA AACT, two contigs/unigenes for HMGS, six contigs/unigenes for HMGR, two contigs/unigenes for MVK, two contigs/unigenes for PMVK, and three contigs/unigenes for MVD. 38 up-regulated unigenes among 49 DEGs were related to methylerythritol phosphate (MEP) pathway or 1-deoxy-D-xylulose-5-phosphate (DXP) pathway, including five contigs/unigenes for DXPS, three contigs/ unigenes for DXPR, four contigs/unigenes for 4hydroxy-3-methylbut-2-(E)-enyl-diphosphate synthase (ispG), three contigs/unigenes for IDI, 12 contigs/unigenes for isoprene synthase, and 11 contigs/unigenes for FPPS. These contigs/unigenes might participate in the biosynthesis of IPP that is the building block of terpenoids (Table 5).

\section{Identification of sesquiterpene biosynthetic genes}

According to the KEGG annotation, there are 75 DEGs correlated with sesquiterpenoid and triterpenoid biosynthesis, accounting for $0.63 \%$ among 11,948 DEGs. 22 DEGs related to sesquiterpenoid biosynthesis were listed in Additional file 5, including 13 up-regulated and nine down-regulated unigenes, and involved metabolic pathways were shown in Additional file 4. These DEGs included genes encoding acyclic sesquiterpenoid synthase, bisabolene-type synthase, germacren-type synthase, humulene-type synthase and cadinyl-type synthase. Among all five types of sequiterpene synthases, the germacrene-type synthases have interested us. There are four types of germacrene-type synthases, including germacrene D synthases, germacradienol synthases, valencene synthases and 7-epi-alpha-selinene synthases. Totally, ten unigenes, including CL7773.Contig1_All, CL7773.Contig2_All, CL7773.Contig3_All, CL7773.Contig4_All, CL7773.Contig5_All, CL7773.Contig8_All, CL8776.Contig1_All, CL8776.Contig2_All, CL9179.Contig2_All, Unigene46663_All, encoding germacrene D synthases and germacradienol synthases expressed higher in roots and four unigenes, including CL12078. Contig1_All, Unigene4834_All, CL12078.Contig2, CL12078.Contig3_All, expressed higher in leaves. One unigene CL7773.Contig8_All encoding valencene synthase and 7-epi-alpha-selinene synthase expressed higher in roots and no unigenes expressed higher in leaves. CL7773.Contig8_All was annotated in all four germacrene-type synthases (Additional file 5). Besides, we performed phylogenetic analysis for the unigenes with representative plant sequiterpene synthases. The phylogenetic analysis revealed that CL7773.Contig1_All, CL8776.Contig2_All and CL8776.Contig1_All belonged to TPS-a subfamily, while CL9179.Contig2_All, CL7773.Contig4_All, CL7773.Contig2_All and CL7773.Contig5_All belonged to TPS-b subfamily (Fig. 6a). 


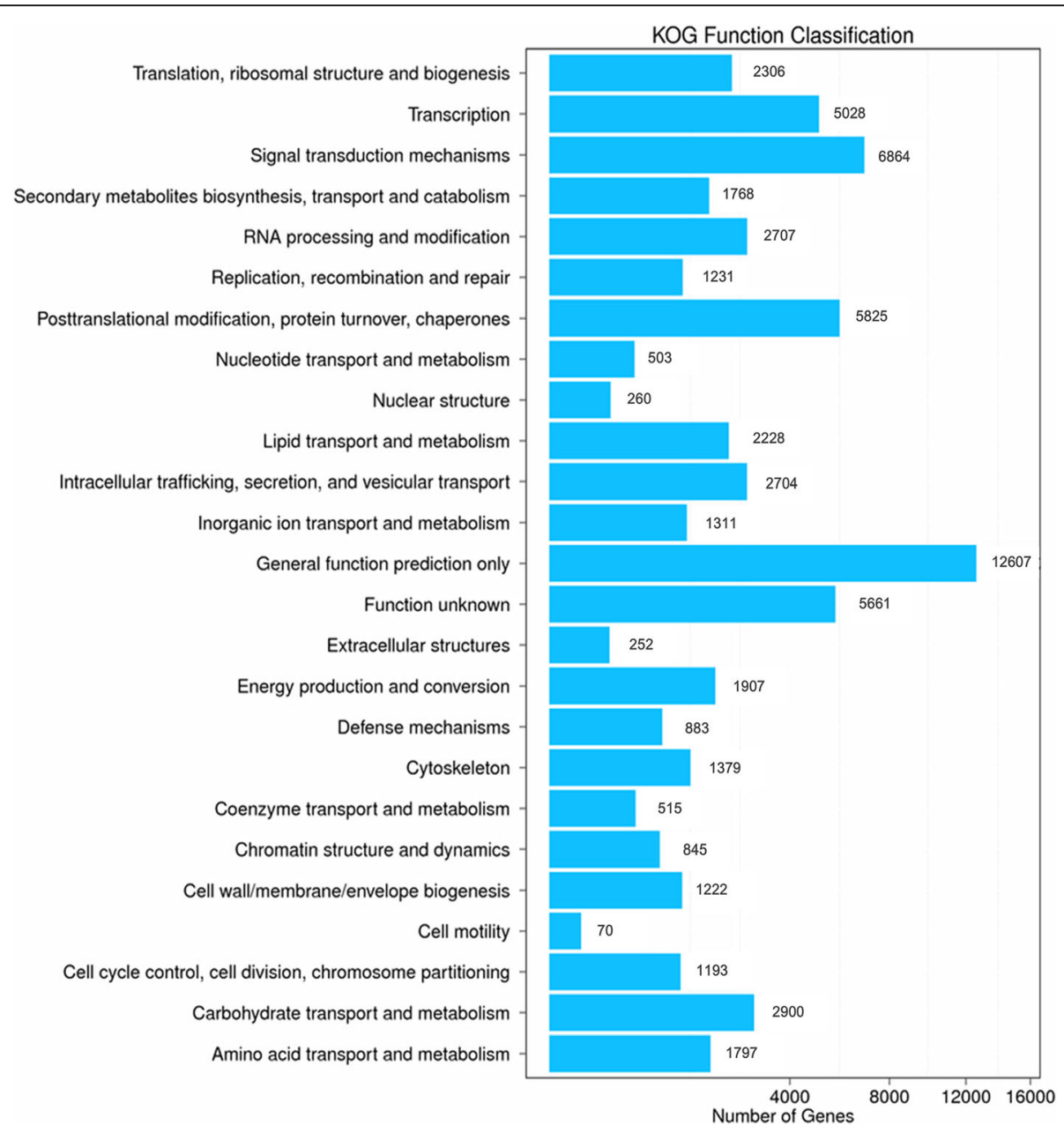

Fig. $3 \mathrm{KOG}$ function classification of $C$. angulatus. A total of 51,817 unigenes were classified into 25 functional categories according to their predicted gene products using the COG database. This figure was generated with R 3.2.5

In addition, in the downstream pathway of Celangulin $\mathrm{V}$ biosynthesis, some functional groups on the sesquiterpene skeleton are needed. Specifically, CYP450s and acyltransferases play major roles in this part. 288 unigenes were identified for the CYP450 family. Among them, 142 DEGs (88 up-regulated and 54 down-regulated unigenes) were discovered. There were 18 unigenes related to CYP 71 clan, including 13 up-regulated and five down-regulated unigenes. The 13 up-regulated unigenes encoding CYP71A1, CYP71A9, CYP71A25, CYP71D9, CYP71D10, CYP71D11, CYP71E7, CYP76A2, CYP83B1. The CYP71D9, CYP71D10, CYP71D11 have interested us, including CL8302.Contig2_All, CL12402. Contig1_All, Unigene922_All, CL5925.Contig2_All, CL12355.Contig1_All, CL12355.Contig4_All, CL5885. Contig1_All, CL5885.Contig2_All. (Additional file 6). A phylogenetic analysis of the eight unigenes with 44 plant cytochrome P450s involved in terpenoids biosynthesis including gibberellins, abscisic acid, carotenoids and plant defense substances. The phylogenetic analysis of
CYPs revealed that all of the eight unigenes belonged to the CYP71D subfamily (Fig. 6b).

Two hundred thirty seven unigenes were related to acyltransferase family. Among them, 60 DEGs (33 up-regulated and 27 down-regulated unigenes) were discovered. There were four unigenes related to a plant acyl-CoA dependent acyltransferase superfamily, BAHD, including CL5679.Contig1_All, CL5679.Contig9_All, Unigene21128_All and CL2580.Contig2_All expressed higher in roots (Table 5).

\section{Validation and expression analysis of key genes}

To confirm the accuracy of the BGISEQ-500 sequencing and FPKM calculated results, we selected 15 unigenes and used qRT-PCR to determine their relative expression level in the leaf and root tissues of $C$. angulatus. All 15 unigenes were putative sesquiterpene biosynthetic genes containing five higher-expressed unigenes (CL1103.Contig4_All, CL5925.Contig1_All, Unigene27813_All, CL1173.Contig1_All, and Unigene225446_All), five lower-expressed 


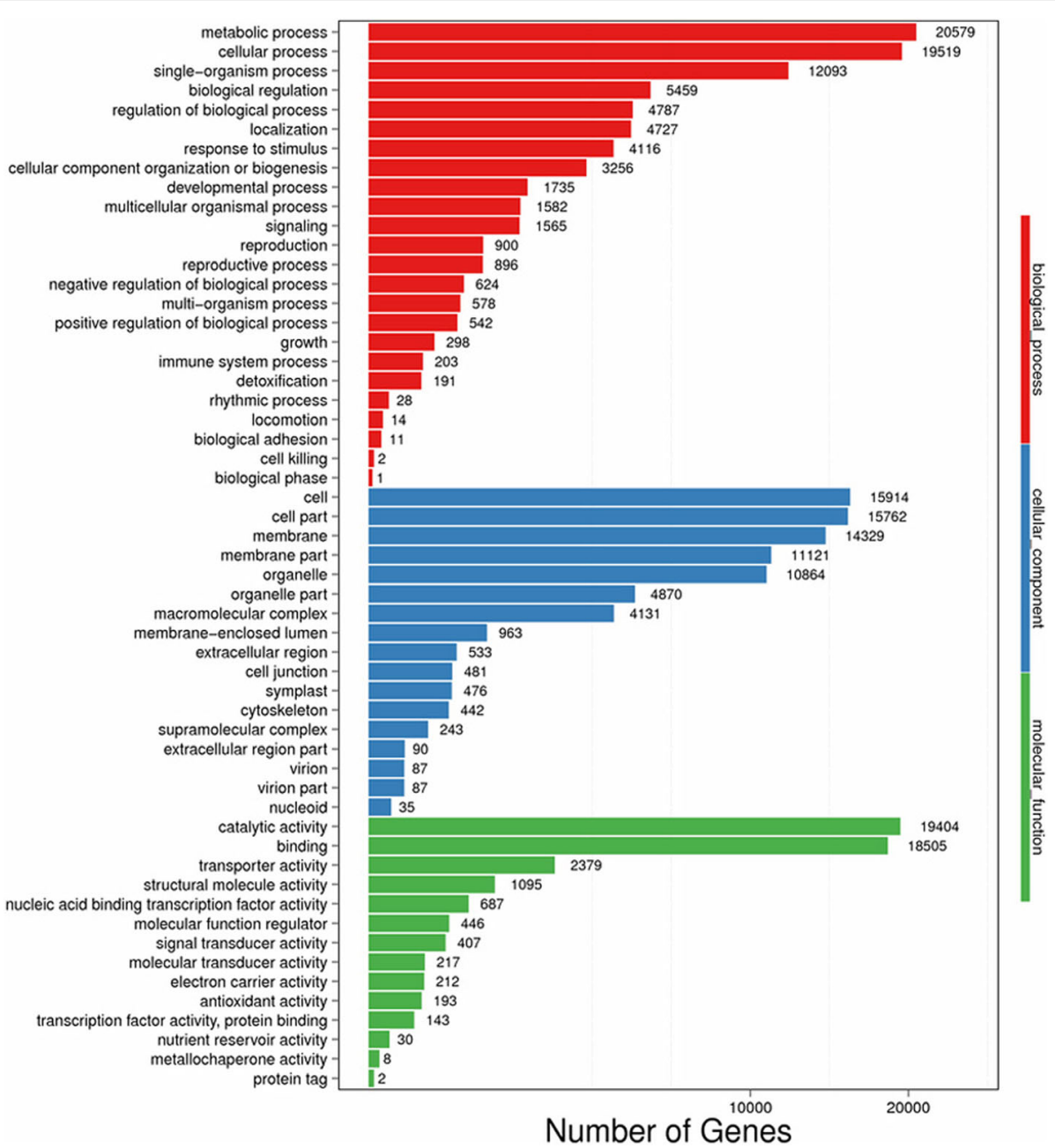

Fig. $4 \mathrm{GO}$ categories of $C$. angulatus. The results are summarized in mainly three categories: biological process, cellular component and molecular function. This figure was generated with R 3.2.5

unigenes (Unigene16075_All, Unigene15369_All, CL4937. Contig4_All, CL4195.Contig1_All, and CL5143.Contig3_All) and five unchanged unigenes (CL7270.Contig2_All, Unigene19019_All, CL674.Contig2_All, CL270.Contig12_All, and Unigene9812_All) calculated by FPKM. The qRT-PCR and FPKM results were shown in Fig. 7, and the expression levels are similar.

\section{Discussion}

\section{BGISEQ-500 sequencing and sequence annotation}

C. angulatus is a kind of excellent traditional insecticidal plant due to their ample active ingredients of sesquiterpenes ( $\beta$-agarofurans), alkaloids and flavonoids. Even though Celangulin V is considered as the most vital active constituents of $C$. angulatus, little is known about the biosynthetic and metabolic mechanisms of this sesquiterpene polyol ester. The aims of this study were to generate a large amount of cDNA sequence data that would facilitate more detailed studies on $C$. angulatus, and to identify the genes related to sesquiterpene polyol esters biosynthesis and metabolism. The availability of transcriptome data for $C$. angulatus will meet the initial information needs for functional studies of this species and its relatives. Six RNA-seqs were performed using BGISEQ-500 sequencing, which generated a total of 104,950 unigenes. 71,479 (68.11\%) unigenes provided a significant BLAST result. This is the first report about transcriptome study of $C$. angulatus, providing adequate references to study on other plants with close relationship to $C$. angulatus.

\section{Terpenoid backbone biosynthetic genes and their differential expression patterns in C. angulatus}

In previous studies on $C$. angulatus, Celangulin $\mathrm{V}$ were shown to be distributed in many organs, but mainly accumulated in root bark, the contents of Celangulin V showed higher in root than in leaf, and changed in different developmental stages [5-7]. We speculated that 
Table 4 Pathways and number of unigenes related to secondary metabolites in C. angulatus

\begin{tabular}{lll}
\hline $\begin{array}{l}\text { Biosynthesis of secondary metabolites } \\
\text { pathway }\end{array}$ & Pathway ID & $\begin{array}{l}\text { All genes with } \\
\text { pathway } \\
\text { annotation } \\
(48,810)\end{array}$ \\
\hline Anthocyanin biosynthesis & ko00942 & $29(0.06 \%)$ \\
Benzoxazinoid biosynthesis & ko00402 & $30(0.06 \%)$ \\
Betalain biosynthesis & ko00965 & $5(0.01 \%)$ \\
Brassinosteroid biosynthesis & ko00905 & $54(0.11 \%)$ \\
Caffeine metabolism & ko00232 & $16(0.03 \%)$ \\
Carotenoid biosynthesis & ko00906 & $198(0.41 \%)$ \\
Cyanoamino acid metabolism & ko00460 & $314(0.64 \%)$ \\
Diterpenoid biosynthesis & ko00904 & $112(0.23 \%)$ \\
Flavone and flavonol biosynthesis & ko00944 & $46(0.09 \%)$ \\
Flavonoid biosynthesis & ko00941 & $231(0.47 \%)$ \\
Indole alkaloid biosynthesis & ko00901 & $93(0.19 \%)$ \\
Isoquinoline alkaloid biosynthesis & ko00950 & $126(0.26 \%)$ \\
Monoterpenoid biosynthesis & ko00902 & $49(0.1 \%)$ \\
Monobactam biosynthesis & ko00261 & $67(0.14 \%)$ \\
Nicotinate and nicotinamide & ko00760 & $181(0.37 \%)$ \\
metabolism & & $687(1.41 \%)$ \\
Phenylpropanoid biosynthesis & ko00940 & $145(0.3 \%)$ \\
Sesquiterpenoid and triterpenoid & ko00909 & $225(0.46 \%)$ \\
Siosynthesis & & $175(0.36 \%)$ \\
Stilbenoid, diarylheptanoid and & ko00100 & $310(0.64 \%)$ \\
Terpenoid backbone biosynthesis & ko00900 & $142(0.29 \%)$ \\
Tropane, piperidine and pyridine & ko00960 & \\
\hline & & \\
\hline lkaloid biosynthesis & & \\
\hline
\end{tabular}

the genes expressed higher in root might encode some enzymes responsible for the biosynthesis of Celangulin V. Therefore, choosing the root and the leaf for comparative transcriptome analysis will greatly facilitate dissection of the genes involved in the organ-specific biosynthesis of Celangulin V. This approach is widely used for mining and identifying novel genes in biosynthesis of secondary metabolites in plants [35-37].

IPP and its isomer DMAPP, the universal biological precursors of all isoprenoids (basic C5 isoprene unit), can be obtained either through MVA/MEP pathways [38] (Fig. 1) in all eukaryotic cells and cytoplasm and mitochondria of plants or MEP pathway in bacteria, other prokaryotes and plastids in plants [39].

Totally, 22 up-regulated unigenes related to MVA pathway and 38 up-regulated unigenes related to MEP pathway possibly involved in terpenoid backbone biosynthesis are listed in Table 5. Numerous studies have been conducted with regard to engineer microorganisms for the production of different isoprenoids. The yeast Saccharomyces cerevisiae was always chosen as a microbial host to enhance the production of sesquiterpene by deregulating the MVA pathway, and the Escherichia coli was usually chosen as the microbial host for deregulating the MEP pathway. For example, the production of artemisinic acid engineered by MVA pathway achieved nearly 500 times production as previously reported [40]. Other natural products, such as patchoulol, farnesol, limonene and lycopene have also got higher titers through MEP or MVA metabolic pathway engineering methods [38, 41, 42]. In this study, the 22 up-regulated genes involved in MVA pathway and 38 up-regulated genes involved in MEP pathway were the candidate genes that can enhance the availability of the precursor (FPP) for biosynthesis of sesquiterpenes, and have the potential to improve the production of Celangulin V.
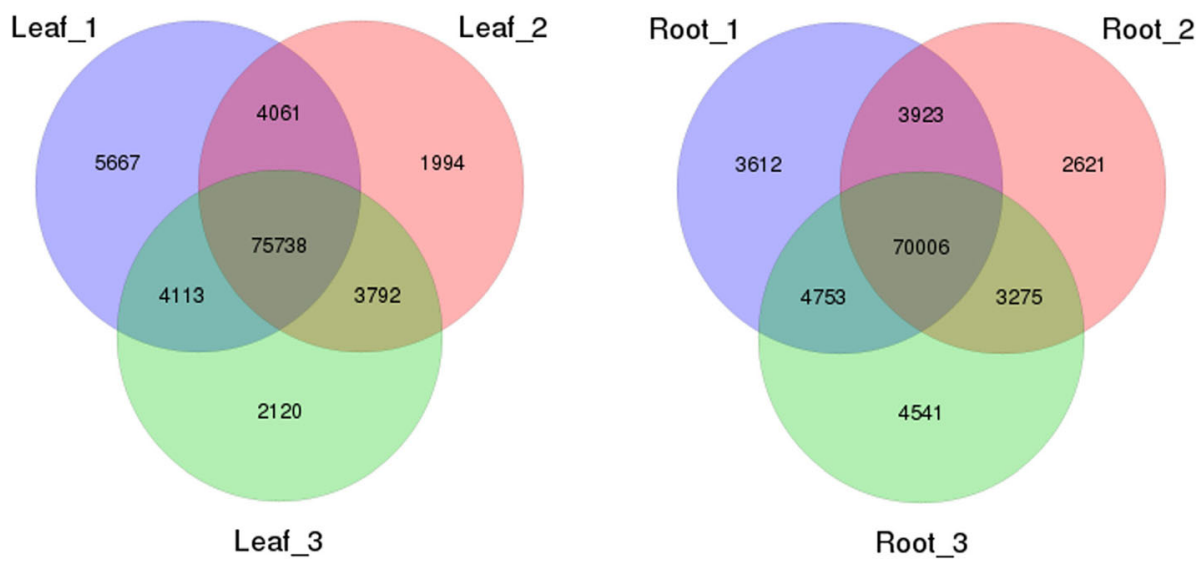

Fig. 5 Venn diagram of the unigenes at Leaf and Root. This figure was generated with R 3.2.5 
Table 5 Discovery and expression of unigenes involved in celangulin V biosynthesis in C. angulatus

\begin{tabular}{|c|c|c|c|}
\hline Enzymes name & Abbreviation & EC number & Expressed higher \\
\hline Acetyl-CoA C-acetyltransferase & AACT & EC:2.3.1.9 & $\begin{array}{l}\text { CL9396.Contig2_All, Unigene26040_All, Unigene13706_All, } \\
\text { CL270.Contig9_All, CL270.Contig8_All, CL270.Contig11_All, } \\
\text { Unigene1229_All }\end{array}$ \\
\hline Hydroxymethylglutaryl-CoA synthase, & HMGS & $E C: 2.3 .3 .10$ & CL1173.Contig1_All, CL1173.Contig2_All \\
\hline Hydroxymethylglutaryl-CoA reductase & HMGR & EC:1.1.1.34 & $\begin{array}{l}\text { CL3155.Contig6_All, CL7171.Contig2_All, Unigene9669_All, } \\
\text { Unigene28303_All, CL3155.Contig5_All, CL3155.Contig2_All }\end{array}$ \\
\hline Mevalonate kinase & MVK & $E C: 2.7 .1 .36$ & CL11783.Contig1_All, CL11783.Contig2_All \\
\hline Phosphomevalonate kinase & PMVK & EC:2.7.4.2 & CL8165.Contig1_All, Unigene10676_All \\
\hline Diphosphomevalonate decarboxylase & - & EC:4.1.1.33 & CL9292.Contig1_All, Unigene24911_All, Unigene27813_All \\
\hline 1-deoxy-D-xylulose-5-phosphate synthase & DXPS & EC:2.2.1.7 & $\begin{array}{l}\text { CL4067.Contig9_All, CL4067.Contig8_All, CL4067.Contig7_All, } \\
\text { CL4067.Contig5_All, Unigene2397_All }\end{array}$ \\
\hline $\begin{array}{l}\text { 1-deoxy-D-xylulose-5-phosphate } \\
\text { reductoisomerase }\end{array}$ & DXPR & EC:1.1.1.267 & CL10481.Contig1_All, Unigene29109_All, CL10481.Contig2_All \\
\hline $\begin{array}{l}\text { 4-hydroxy-3-methylbut-2-(E)-enyl-diphosphate } \\
\text { synthase }\end{array}$ & ispG & EC:1.17.7.1 1.17.7.3 & $\begin{array}{l}\text { Unigene27344_All, CL2211.Contig12_All, CL2211.Contig16_All, } \\
\text { CL2211.Contig17_All }\end{array}$ \\
\hline $\begin{array}{l}\text { 4-hydroxy-3-methylbut-2-en-1-yl diphosphate } \\
\text { reductase }\end{array}$ & ispH & EC:1.17.7.4 & - \\
\hline isopentenyl-diphosphate $\delta$-isomerase & $|\mathrm{D}|$ & EC:5.3.3.2 & CL674.Contig5_All, CL39.Contig4_All, CL674.Contig3_All \\
\hline isoprene synthase & - & EC:4.2.3.27 & $\begin{array}{l}\text { CL4994.Contig7_All, CL4994.Contig2_All, CL4994.Contig6_All, } \\
\text { CL4994.Contig8_All, CL4994.Contig9_All, CL686.Contig1_All, } \\
\text { CL4994.Contig1_All, CL4994.Contig3_All, CL4994.Contig10_All, } \\
\text { CL686.Contig2_All, CL4994.Contig4_All, Unigene2738_All }\end{array}$ \\
\hline Farnesyl diphosphate synthase & FPP & EC:2.5.1.1 2.5.1.10 & $\begin{array}{l}\text { Unigene5517_All, Unigene20963_All, Unigene18861_All, } \\
\text { Unigene28032_All, Unigene8091_All, Unigene43426_All, } \\
\text { CL6922.Contig1_All, CL2794.Contig15_All, CL2794.Contig18_All, } \\
\text { CL2794.Contig3_All, CL2794.Contig12_All }\end{array}$ \\
\hline $\begin{array}{l}\text { Germacrene D synthase } \\
\text { Germacradienol synthase }\end{array}$ & - & $\begin{array}{l}E C: 4 \cdot 2 \cdot 3 \cdot 75 \\
\text { EC:4.2.3.22 }\end{array}$ & CL7773.Contig1_All, CL8776.Contig2_All, CL8776.Contig1_All, \\
\hline Valencene/7-epi-alpha-selinene synthase & - & EC:4.2.3.73 4.2.3.86 & CL7773.Contig8_All \\
\hline CYP71D9/CYP71D10/CYP71D11 & - & $\begin{array}{l}\text { EC: } 1.14 .14 .1 \\
\text { EC:1.14.14.19 } \\
1.14 .14 .32\end{array}$ & $\begin{array}{l}\text { CL8302.Contig2_All, CL12402.Contig1_All, Unigene922_All, } \\
\text { CL5925.Contig2_All, CL12355.Contig1_All, CL12355.Contig4_All, } \\
\text { CL5885.Contig1_All, CL5885.Contig2_All, }\end{array}$ \\
\hline BAHD acyltransferase & - & $\begin{array}{l}\text { EC:2.3.1.- } \\
\text { EC:2.3.1.133 }\end{array}$ & $\begin{array}{l}\text { CL5679.Contig1_All, CL5679.Contig9_All } \\
\text { Unigene21128_All, CL2580.Contig2_All }\end{array}$ \\
\hline
\end{tabular}

\section{Sesquiterpene biosynthetic genes and their differential expression patterns in $C$. angulatus}

Terpenes, the largest class of natural products, are formed by terpene cyclases from linear oligoprenyl diphosphate precursors [43]. The phylogentic analyses of plant TPS protein sequences recognized seven major clades or subfamilies (TPS-a, TPS-b, TPS-c, TPS-d, TPS-e/f, TPS-g and TPS-h) $[44,45]$. C. angulatus belongs to angiosperms. TPSs involved in angiosperm sesquiterpene synthesis are mainly distributed in TPS-a subfamily, with a small part dispersed in TPS-g/h subfamily. However, those involved in angiosperm monoterpene synthesis are mainly distributed in TPS-b subfamily [46]. CL7773.Contig1_All, CL8776.Contig2_All and CL8776.Contig1_All fell into TPS-a subfamily according to the phylogenetic analysis. Therefore, these unigenes maybe the sesquiterpene synthases and are predicted to be germacrene D synthase and germacradienol synthase [47, 48]. The CL7773.Contig8_All is predicted to be valencene/7-epi-alpha-selinene synthase, and the recombinant enzyme from Vitis vinifera gave 49.5\% (+)-valencene and $35.5 \%(-)-7$-epi- $\alpha$-selinene [47]. These four unigenes maybe involved in the Celangulin V biosynthesis (Table 5).

Many studies have proposed the cyclization mechanisms of sesquiterpene synthases $[49,50]$. The different cyclization processes can form diverse structures [51]. FPP cyclization reactions can proceed through $\mathrm{C} 1-\mathrm{C} 6$, $\mathrm{C} 1-\mathrm{C} 7, \mathrm{C} 1-\mathrm{C} 10$ and $\mathrm{C} 1-\mathrm{C} 11$ bond formation depending on which carbon-carbon double bond reacts with the initially formed allylic carbocation [52]. Celangulin V is a sesquiterpene polyol ester with $\beta$-dihydroagarofuran skeleton. Based on the chemical structural characteristics of $\beta$-dihydroagarofuran, we speculated that these four unigenes encoding sesquiterpene synthases could convert FPP to $\beta$-dihydroagarofuran through $\mathrm{C} 1-\mathrm{C} 10$ bond formation. In the future research, we will identify the function of candidate genes for sesquiterpene 


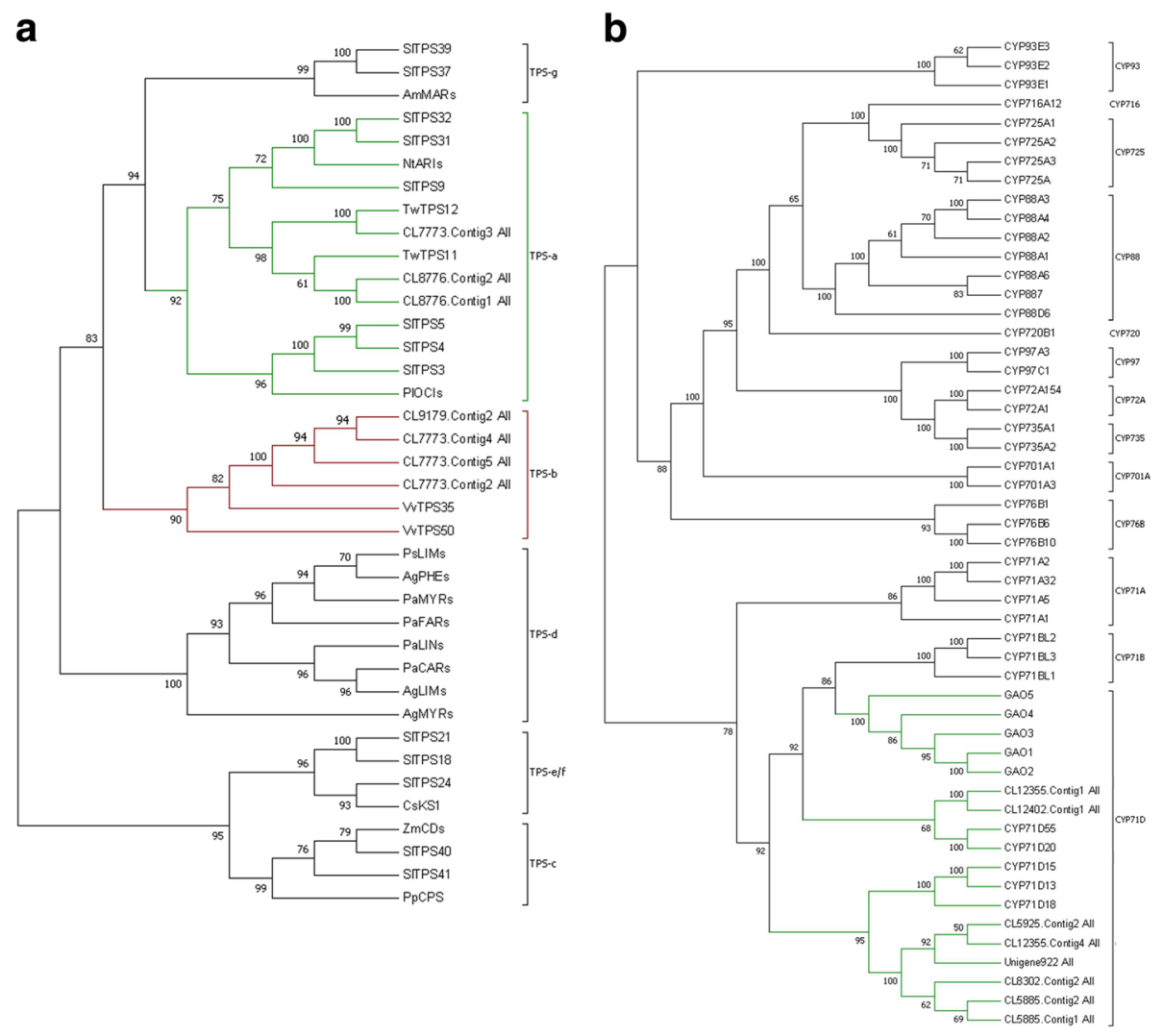

Fig. 6 Phylogenetic analysis of terpene synthase, CYP450. a Phylogenetic tree of terpene synthase nucleotide sequence. $\mathbf{b}$ Phylogenetic tree of CYP450 nucleotide sequence. Accession numbers of proteins used in phylogenetic analysis are listed in Additional file 7 . This figure was generated with MEGA 7.0.26

synthases belonging to the Celangulin $\mathrm{V}$ biosynthetic pathway by experiment.

\section{Celangulin $\mathrm{V}$ biosynthesis modified genes and their differential expression patterns in $C$. angulatus}

Plant CYP450s [53-55] and acyltransferases [56] modify the sesquiterpene carbon ring and are important in biotechnology due to their ability to biosynthesize diverse secondary metabolites with various biological properties. The CYP71 clan is the largest P450 family and functionally diverse in all plant species [54], including a total of 110 members divided into 18 subfamilies and showing large clusters of duplicated genes and taxa-specific subfamily blooms [57]. The CYP71 clan is also called the cradle of monoterpenoid and sesquiterpenoid diversity. P450s involved in sesquiterpenoids metabolism have spawned exclusively in five families of the CYP71 clan: CYP71AV, CYP71BA, CYP71BL, CYP71D, CYP706B [58]. So far, the functions of many CYP450s have been identified, participating in the biosynthesis of terpenoid-based natural products in various plants [54]. At present, six bioactive sesquiterpenes have been successfully produced in heterologous systems by using CYP450 coding sequences
[53]. In the capsidiol biosynthesis, 5-epi-aristolochene 1,3-dihydroxylase (CYP71D20) played a decisive role and the preferred reaction order of hydroxylation is at $\mathrm{C} 1$ followed by C3 [59]; CYP706B1 (part of the CYP71 clan) catalyzed hydroxylation of $\delta$-cadinene at $\mathrm{C} 8$ in gossypol's biosynthesis [60]; CYP71BA1 from Zingiber zerumbet (Zingiberaceae) catalyzed the conversion of a-humulene to 8-hydroxy-a-humulene at $\mathrm{C} 8$ in the zerumbone biosynthesis [61]; CYP71BL2 from Lactuca sativa [62] and CYP71BL3 from Cichorium intybus [63] specifically catalyzed 6a-hydroxylation by mediate hydroxylation of germacrene A acid at positions adjacent to the carboxy group. We obtained eight unigenes that were predicted to be the CYP71D9, CYP71D10, CYP71D11 may involve in Celangulin V biosynthesis (Table 5).

It is not well documented that acyltransferases are recruited as catalysts in the biosynthesis of plant specialized sesquiterpenoid compounds. Take artemisinin [40] and capsidiol [64] for examples, the acylated groups are not needed in their biosynthesis. However, some plant terpenoids need an acylation post-modification, which is depend on BAHD superfamily acyltransferases [65]. The BAHD acyltransferases are named after the first four 

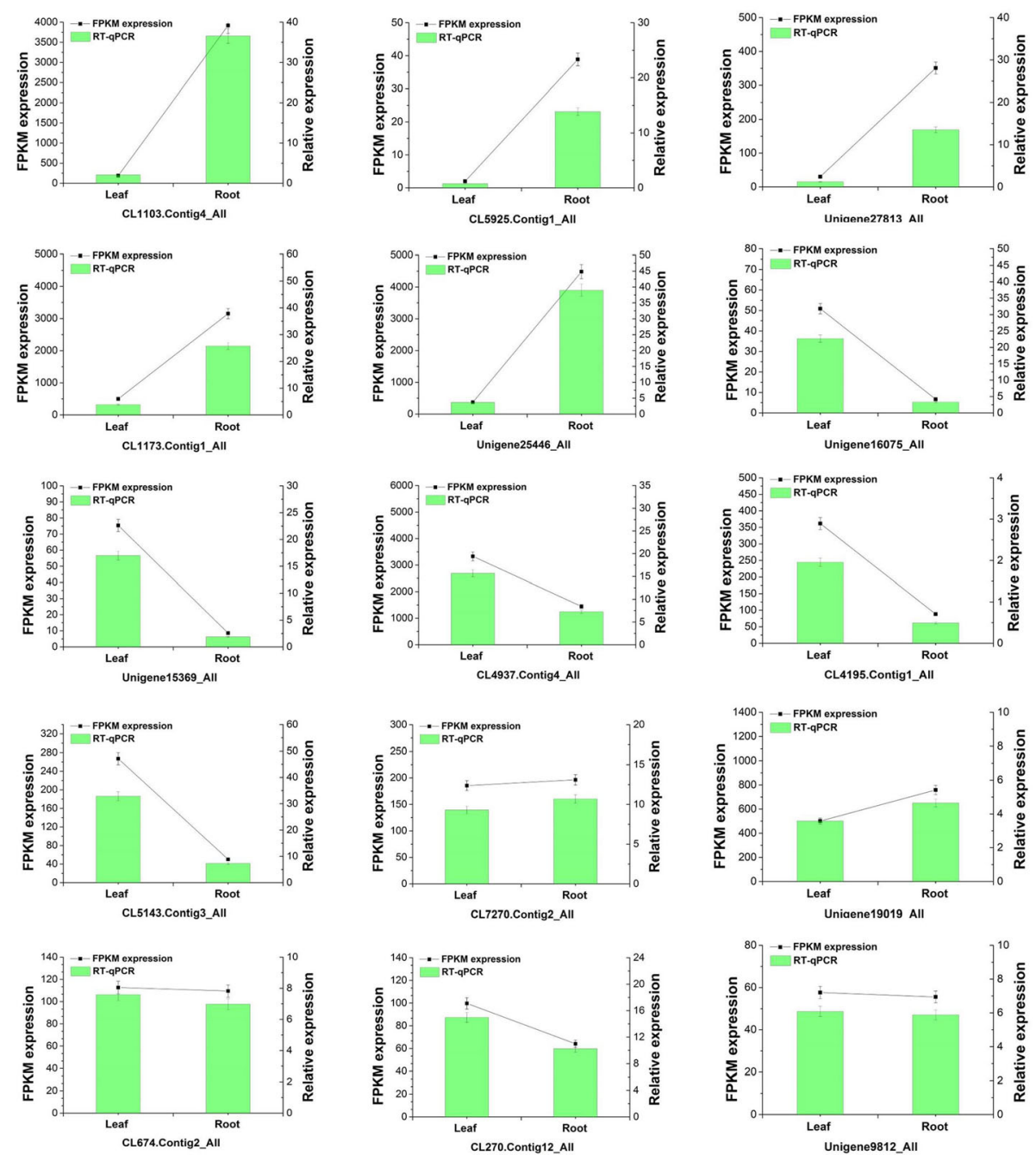

Fig. 7 qRT-PCR validation of selected 15 DEGs at Leaf and Root. The relative expression level of each selected gene was determined by $2-\Delta \Delta C T$. Each bar represents the mean \pm STD of triplicate assays. Values with different letters indicate significant differences at $P<0.05$ according to Duncan's multiple range tests. This figure was generated with OriginPro 8.5.1SR2

biochemically characterized enzymes of the group, which use acyl-CoA thioesters as donor molecules [66]. For example, two BAHD alcohol acetyltransferases were identified from $L . x$ intermedia glandular trichome database, which convert a variety of monoterpenes to monoterpene esters using coenzyme A as a cofactor [67]. Several taxoid-O-acetyl transferases (like TAX 9 and TAX 14), obtained from a previously isolated family of Taxus acyl/ aroyl transferase cDNA clones, could catalyze some acylation steps of Taxol biosynthesis $[68,69]$. We obtained four unigenes that were predicted to be the BAHD acyltransferase from transcriptome data, probably participating in biosynthesis of Celangulin $\mathrm{V}$, according to acylated groups on $\mathrm{C} 5, \mathrm{C} 6, \mathrm{C} 8, \mathrm{C} 9$ and $\mathrm{C} 14$ positions of $\beta$-dihydroagarofuran skeleton.

Accordingly, we proposed the biosynthetic pathway of Celangulin V: $\beta$-dihydroagarofuran is the parent skeleton, highly oxygenated at C1, C3, C5, C6, C8, C9, C14 sites by one or more CYP450s to produce dihydroagarofuran polyols; Then, C5, C6 hydroxyl groups are acetylated by acetyltransferases; C9, C14 hydroxyl groups are butyrylated by butyryltransferase; $\mathrm{C} 8$ hydroxyl group is benzoylated by benzoyl transferase; C1, C3 are free. Based on our transcriptome sequencing, we annotated eight up-regulated CYP450 unigenes and four up-regulated BAHD acyltransferases in the sesquiterpene biosynthesis. In the future research, we will identify the function of candidate CYP450 and acyltransferase genes.

\section{Conclusions}

Celangulin $\mathrm{V}$ is an efficient insecticidal sesquiterpenoid and widely used in pest control in China. In this study, leaf and root transcriptome were generated by next -generation sequencing, obtaining 104,950 unigenes with 
average length of $1200 \mathrm{bp}$. The transcriptome analysis revealed 16 unigenes probably involved in Celangulin V biosynthesis, in which four unigenes encoded sesquiterpene synthases (germacrene D synthase, germacradienol synthase, valencene synthase and 7-epi-alpha-selinene synthase), eight unigenes encoded CYP450s (CYP71D9, CYP71D10, CYP71D11) and four unigenes encoded BAHD acyltransferases. We further proposed the Celangulin V biosynthetic pathway. The transcriptome data not only provides valuable information on sesquiterpene polyol ester with $\beta$-dihydroagarofuran skeleton but also assists in mining genes involved in the production of other plants with close relationship to C. angulatus.

\section{Additional files}

Additional file 1: The candidate transcript sequences that are involved in the biosynthesis of Celangulin V. A total of 16 unigenes were involved in the biosynthesis of Celangulin $\mathrm{V}$, in which four unigenes encoded sesquiterpene synthases, eight unigenes encoded CYP450s and four unigenes encoded BAHD acyltransferases. (DOCX $22 \mathrm{~kb}$ )

Additional file 2: Genes and primers used for qRT-PCR analysis. Primers were designed using the Primer Premier program (version 5.0). (XLSX $22 \mathrm{~kb}$ )

Additional file 3: Summary of functional annotations of $C$. angulatus. The number of transcripts which be annotated with at least one functional database. (DOCX $15 \mathrm{~kb}$ )

Additional file 4: Putative Sesquiterpenoid and triterpenoid biosynthesis pathway of $C$. angulatus. Putative Sesquiterpenoid and triterpenoid biosynthesis of $C$. angulatus was constructed based on KEGG annotation. A total of 75 unigenes were involved in the metabolic pathway. These unigenes were distributed in the rectangular boxes in the figure. (DOCX $41 \mathrm{~kb}$ )

Additional file 5: 30 DEGs involved sesquiterpenoid biosynthesis in $C$. angulatus. The DEGs were assigned to KEGG biochemical pathways in sesquiterpenoid biosynthesis. (DOCX $17 \mathrm{~kb}$ )

Additional file 6: Summary of unigenes annotated as CYP450 and Acyltransferase. The details of CYP450 family, DEGs of CYP450 family, CYP71 Clan, Acyltransferase family, DEG of Acyltransferase family and BAHD Acyltransferases were showed in XLSX document. (XLSX $140 \mathrm{~kb}$ )

Additional file 7: Accession numbers of terpene synthase and CYP450 proteins used in the phylogenetic analysis. (DOCX $25 \mathrm{~kb}$ )

\section{Abbreviations}

AACT: C-acetyltransferase; CMK: 4-(cytidine 5'-diphospho)-2-C-methyl-Derythritol kinase; DEGs: Differentially expressed genes; DMAPP: Dimethylallyl diphosphate; DXP: 1-deoxy-D-xylulose-5-phosphate; DXPR: 1-deoxy-Dxylulose-5-phosphate reductoisomerase; DXPS: 1-deoxy-D-xylulose-5phosphate synthase; FDR: False discovery rate; FPKM: Fragments per kilobase per transcript per million mapped reads; FPP: Farnesyl diphosphate; FPPS: Farnesyl diphosphate synthase; GO: Gene ontology; GPPS: Geranyl diphosphate synthase; HDR: (E)-4-hydroxy-3-methylbut-2-enyl diphosphatereductase; HDS: (E)-4-hydroxy-3-methylbut-2-enyl diphosphate synthase; HMGR: Hydroxymethylglutaryl-CoA reductase:

HMGS: Hydroxymethylglutaryl-CoA synthase; IDI: Isopentenyl-diphosphate $\delta$ isomerase; IPP: Isopentenyl diphosphate; ispG: 4-hydroxy-3-methylbut-2-(E)enyl-diphosphate synthase; KEGG: Kyoto Encyclopedia of Genes and Genomes protein databases; KO: KEGG ortholog database; KOG: Clusters of euKaryotic Orthologous Group database; MCT: 2-C-methyl-D-erythritol 4phosphate cytidylyltransferase; MDS: 2-C-methyl- D -erythritol 2,4cyclodiphosphate synthase; MEP: 2-methylerythritol 4-phosphate pathways; MEP: Methylerythritol phosphate; MVA: Mevalonate; MVD: Mevalonate diphosphate decarboxylase; MVK: Mevalonate kinase; NR: NCBI nonredundant protein database; NT: NCBI non-redundant nucleotide sequence
}

database; PMVK: Phosphomevalonate kinase; qRT-PCR: Quantitative Real-Time PCR; RIN: RNA Integrity Number

\section{Acknowledgements}

Not applicable.

\section{Funding}

This work was supported by National Key R\&D Program of China (2017YFD0201400). The funders did not have any role in the design of the study, collection, analysis, or interpretation of data or in the writing of the manuscript.

\section{Availability of data and materials}

All data generated or analyzed during the current study are included in this article and its supplementary information files. The raw reads have been deposited in the NCBI Sequence Read Archive (SRA) database and the accessions for our submission are STUDY: PRJNA509994, SAMPLE: Root (SAMN10592333) and Leaf (SAMN10592332), EXPERIMENT: Root_1, Root_2, Root_3 (SRX5129236) and Leaf_1, Leaf_2, Leaf_3 (SRX5129237), RUN: Root_1_1.fq.gz (SRR8316533) and Leaf_1_1.fq.gz (SRR8316532). These datasets were derived from the following public domain resources: https:// www.ncbi.nlm.nih.gov/sra/?term=PRJNA509994.

\section{Authors' contributions}

$W L, X Y, J Q, Q C, G Z$ and $W X$ conceived and designed the experiment; $Z H, R X$, $\mathrm{LZ}$ and $\mathrm{XQ}$ collected plant samples and extracted RNA for sequencing; $\mathrm{WL}$, $X Y$ and $D L$ analyzed RNA-Seq data; $W L, X Y$ and DL wrote and modified the manuscript. All authors read and approved the final manuscript.

\section{Ethics approval and consent to participate}

The locations of material collected here are neither privately owned lands nor protected areas. No specific permits were required for our research.

\section{Consent for publication}

Not applicable.

\section{Competing interests}

The authors declare that they have no competing interests.

\section{Publisher's Note}

Springer Nature remains neutral with regard to jurisdictional claims in published maps and institutional affiliations.

\section{Author details}

'Department of Pharmaceutical Engineering, School of Chemical Engineering and Technology, Tianjin University, Tianjin 300072, People's Republic of China. ${ }^{2}$ Key Laboratory of Systems Bioengineering (Ministry of Education), Tianjin University, Tianjin 300072, People's Republic of China. ${ }^{3}$ SynBio Research Platform, Collaborative Innovation Center of Chemical Science and Engineering (Tianjin), Tianjin 300072, People's Republic of China. ${ }^{4}$ College of Plant Protection, Institute of Pesticide Science, Northwest A\&F University, Yangling, Shaanxi 712100, People's Republic of China. ${ }^{5}$ Key Laboratory of Botanical Pesticide R\&D in Shaanxi Province, Yangling, Shaanxi 712100, People's Republic of China.

Received: 22 March 2018 Accepted: 19 December 2018 Published online: 05 January 2019

\section{References}

1. Wakabayashi N, Wu WJ, Waters RM, Redfern RE, Jr GDM, Demilo AB, Lusby WR, Andrzejewski D. Celangulin: a nonalkaloidal insect Antifeedant from Chinese bittersweet, Celastrus angulatus. J Nat Prod. 1988;51(3):537-42.

2. Cheng D, Feng M, Ji Y, Wu W, Hu Z. Effects of Celangulin IV and V FromCelastrus angulatus maxim on $\mathrm{Na}+/ \mathrm{K}+-\mathrm{ATPase}$ activities of the oriental armyworm (Lepidoptera: Noctuidae). J Insect Sci. 2016;16(1):59.

3. Zhang J, Cui L, Li L, Hu Z, Zhang Q, Hu Z, Wu W. Synthesis and insecticidal activities of novel nitrogenous derivatives of celangulin-V. Nat Prod Commun. 2014;9(6):745.

4. Zhang HY, Zhao TZ, Dong JJ, Chen RF, Li ZH, Qin HL. Four new sesquiterpene polyol esters from Celastrus angulatus. Phytochem Lett. 2014; 7(1):101-6. 
5. Wang $M$, Qin $H$, Kong M, Li Y. Insecticidal sesquiterpene polyol ester from Celastrus angulatus. Phytochemistry. 1991;30(12):3931-3.

6. Tu Y, Song Q, Wu X, Huang G, Ma Y, Chen Y. Studies on sesquiterpene constituents from celastrus-angulatus. Acta Chim Sin. 1993;51(4):404-8.

7. Wu W, Wang M, Zhu J, Zhou W, Zhaonong Hu A, Ji Z. Five new insecticidal Sesquiterpenoids from Celastrus angulatus. J Nat Prod. 2001;64(3):364-7.

8. Rohdich F, Lauw S, Kaiser J, Feicht RP, Bacher A, Eisenreich W. Isoprenoid biosynthesis in plants - 2 C -methyl- $d$-erythritol-4-phosphate synthase (IspC protein) of Arabidopsis thaliana. FEBS J. 2006;273(19):4446-58.

9. Leopoldini M, Malaj N, Toscano M, Sindona G, Russo N. On the inhibitor effects of bergamot juice flavonoids binding to the 3-hydroxy-3methylglutaryl-CoA reductase (HMGR) enzyme. J Agric Food Chem. 2010; 58(19):10768-73.

10. Haralampidis K, Trojanowska M, Osbourn AE. Biosynthesis of triterpenoid Saponins in plants. Adv Biochem Eng Biotechnol. 2002;75(75):31.

11. Choi DW, Jung JD, Ha YI, Park HW, Dong SI, Chung HJ, Liu JR. Analysis of transcripts in methyl jasmonate-treated ginseng hairy roots to identify genes involved in the biosynthesis of ginsenosides and other secondary metabolites. Plant Cell Rep. 2005;23(8):557-66.

12. Aharoni A, Jongsma MA, Kim TY, Ri MB, Giri AP, Verstappen FWA, Schwab W, Bouwmeester HJ. Metabolic engineering of Terpenoid biosynthesis in plants. Phytochem Rev. 2006;5(1):49-58.

13. Carelli M, Calderini O. Medicago truncatula CYP716A12 is a multifunctional oxidase involved in the biosynthesis of hemolytic saponins. Plant Cell. 2011; 23(8):3070.

14. Qi Z, Xue X, Wu W, Zhang J, Yang R. Preparation of monoclonal antibody against Celangulin $V$ and Immunolocalization of receptor in the oriental armyworm, Mythimna separata Walker (Lepidoptera: Noctuidae). J Agr Food Chem. 2006;54(20):7600-5.

15. Su XH, Zhang ML, Zhan WH, Huo CH, Shi QW, Gu YC, Kiyota H. Chemical and pharmacological studies of the plants from genus Celastrus. Chem Biodivers. 2009;6(2):146-61.

16. Spivey AC, Weston M, Woodhead S. ChemInform abstract: Celastraceae Sesquiterpenoids: biological activity and synthesis. Chemlnform. 2002; 33(21):no

17. Sun X, Zhou S, Meng F, Liu S. De novo assembly and characterization of the garlic (Allium sativum) bud transcriptome by Illumina sequencing. Plant Cell Rep. 2012:31(10):1823-8.

18. Wang Z, Gerstein M, Snyder M. RNA-Seq: a revolutionary tool for transcriptomics. Nat Rev Genet. 2009;10(1):57.

19. Ji A, Jia J, Xu Z, Li Y, Bi W, Ren F, et al. Transcriptome-guided Mining of Genes Involved in Crocin biosynthesis. Front Plant Sci. 2017;8:518.

20. Li J, Zheng W, Long D, Ding L, Gong A, Xiao C, et al. Correction:De NovoSequencing and assembly analysis of thePseudostellaria heterophyllaTranscriptome. PLoS One. 2016;11(10):e164235.

21. Liu MH, Yang BR, Cheung WF, Yang KY, Zhou HF, Kwok JS, et al. Transcriptome analysis of leaves, roots and flowers of Panax notoginseng identifies genes involved in ginsenoside and alkaloid biosynthesis. BMC Genomics. 2015;16(1):265.

22. Qiao F, Cong H, Jiang X, Wang R, Yin J, Qian D, Wang Z, Nick P. De novo characterization of a Cephalotaxus hainanensis transcriptome and genes related to paclitaxel biosynthesis. PLoS One. 2014;9(9):e106900.

23. Grabherr MG, Haas BJ, Yassour M, Levin JZ, Thompson DA, Amit I, et al. Fulllength transcriptome assembly from RNA-Seq data without a reference genome. Nat Biotechnol. 2011;29(7):644.

24. Pertea G, Huang X, Liang F, Antonescu V, Sultana R, Karamycheva S, et al. TIGR gene indices clustering tools (TGICL): a software system for fast clustering of large EST datasets. Bioinformatics. 2003;19(5):651-2

25. Götz S, Garcíagómez JM, Terol J, Williams TD, Nagaraj SH, Nueda MJ, et al. High-throughput functional annotation and data mining with the Blast2GO suite. Nucleic Acids Res. 2008:36(10):3420.

26. Young MD, Wakefield MJ, Smyth GK, Oshlack A. Gene ontology analysis for RNA-seq: accounting for selection bias. Genome Biol. 2010;11(2):1-12.

27. Langmead B, Salzberg SL. Fast gapped-read alignment with bowtie 2. Nat Methods. 2012;9(4):357-9.

28. Li B, Dewey CN, Li B, Dewey CN. RSEM: accurate transcript quantification from RNA-Seq data with or without a reference genome. Bmc Bioinformatics. 2011;12(1):323.

29. Mortazavi A, Williams BA, Mccue K, Schaeffer L, Wold B. Mapping and quantifying mammalian transcriptomes by RNA-Seq. Nat Methods. 2008;5(7):621.
30. Audic S, Claverie JM. The significance of digital gene expression profiles Genome Res. 1997;7(10):986.

31. Saitou N. The neighbor-joining method: a new method for reconstructing phylogenetic tree. Mol Biol Evol. 1987;4(4):406.

32. Felsenstein J. Confidence limits on phylogenies: an approach using the bootstrap. Evolution. 1985:39(4):783-91.

33. Kumar S, Stecher G, Tamura K. MEGA7: molecular evolutionary genetics analysis version 7.0 for bigger datasets. Mol Biol Evol. 2016;33(7):1870.

34. Wu W. In: Liu J, Zhang C, editors. Research and application of plant pesticide-Celangulin. Beijing: Chemical Industry Press; 2011. p. 224-5.

35. Zheng K, Zhang G, Jiang N, Yang S, Li C, Meng Z, Guo Q, Long G. Analysis of the transcriptome of Marsdenia tenacissima discovers putative polyoxypregnane glycoside biosynthetic genes and genetic markers. Genomics. 2014;104(3):186-93.

36. Sun H, Li F, Xu Z, Sun M, Cong H, Qiao F, Zhong X. De novo leaf and root transcriptome analysis to identify putative genes involved in triterpenoid saponins biosynthesis in Hedera helix L. PLoS One. 2017; 12(8):e182243.

37. Li W, Katin-Grazzini L, Gu X, Wang X, El-Tanbouly R, Yer H, et al. Transcriptome analysis reveals differential gene expression and a possible role of gibberellins in a shade-tolerant mutant of perennial ryegrass. Front Plant Sci. 2017:8:868

38. Asadollahi MA, Maury J, Møller K, Nielsen KF, Schalk M, Clark A, Nielsen J. Production of plant sesquiterpenes in Saccharomyces cerevisiae: effect of ERG9 repression on sesquiterpene biosynthesis. Biotechnol Bioeng. 2008; 99(3):666-77.

39. Tholl D. Terpene synthases and the regulation, diversity and biological roles of terpene metabolism. Curr Opin Plant Biol. 2006;9(3):297-304.

40. Ro D, Paradise EM, Ouellet M, Fisher KJ, Newman KL, Ndungu JM, et al. Production of the antimalarial drug precursor artemisinic acid in engineered yeast. Nature. 2006;440(7086):940-3.

41. Du FL, Yu HL, Xu JH, Li CX. Enhanced limonene production by optimizing the expression of limonene biosynthesis and MEP pathway genes in E. coli. Bioresour Bioprocess. 2014;1(1):10.

42. Wang J, Xiong Z, Li S, Wang Y. Exploiting exogenous MEP pathway genes to improve the downstream isoprenoid pathway effects and enhance isoprenoid production in Escherichia coli. Process Biochem. 2015:50(1):24-32.

43. Baer P, Rabe P, Fischer K, Citron CA, Klapschinski TA, Groll M, Dickschat JS. Induced-fit mechanism in class I terpene Cyclases. Angew Chem Int Ed. 2014;53(29):7652-6.

44. Bohlmann J, Meyer-Gauen G, Croteau R. Plant Terpenoid synthases: molecular biology and phylogenetic analysis. P Natl Acad Sci Usa. 1998; 95(8):4126-33.

45. Martin DM, Fäldt J, Bohlmann J. Functional characterization of nine Norway spruce TPS genes and evolution of gymnosperm terpene synthases of the TPS-d subfamily. Plant Physiol. 2004;135(4):1908-27.

46. Chen F, Tholl D, Bohlmann J, Pichersky E. The family of terpene synthases in plants: a mid-size family of genes for specialized metabolism that is highly diversified throughout the kingdom. Plant J. 2011;66(1):212-29.

47. Lücker J, Bowen P, Bohlmann J. Vitis vinifera terpenoid cyclases: functional identification of two sesquiterpene synthase cDNAs encoding (+)-valencene synthase and (-)-germacrene D synthase and expression of mono- and sesquiterpene synthases in grapevine flowers and berries. Phytochemistry. 2004;65(19):2649-59.

48. Cane DE, Watt RM. Expression and mechanistic analysis of a germacradienol synthase from Streptomyces coelicolor implicated in geosmin biosynthesis. P Natl Acad Sci Usa. 2003;100(4):1547-51.

49. Tao Q, Ma K, Yang Y, Wang K, Chen B, Huang Y, et al. Bioactive Sesquiterpenes from the edible mushroom Flammulina velutipes and their biosynthetic pathway confirmed by genome analysis and chemical evidence. J Org Chem. 2016;81(20):9867-77.

50. Rabe P, Pahirulzaman KAK, Dickschat JS. Structures and biosynthesis of Corvol ethers-Sesquiterpenes from the ActinomyceteKitasatospora setae. Angew Chem Int Ed. 2015;54(20):6041-5.

51. Christianson DW. Structural and chemical biology of Terpenoid Cyclases. Chem Rev. 2017;117(17):11570-648.

52. Cane DE, Yang G, Xue Q, Shim JH. Trichodiene synthase. Substrate specificity and inhibition. Biochemistry. 1995;34(8):2471.

53. Villa-Ruano N, Pacheco-Hernández Y, Lozoya-Gloria E, Castro-Juárez C Mosso-Gonzalez C, Ramirez-Garcia SA. Cytochrome P450 from plants: 
platforms for valuable Phytopharmaceuticals. Trop J Pharm Res. 2015;14(4): $731-42$.

54. Rasool S, Mohamed R. Plant cytochrome P450s: nomenclature and involvement in natural product biosynthesis. Protoplasma. 2016;253(5):1197-209.

55. Takahashi S, Yeo YS, Zhao Y, O'Maille PE, Greenhagen BT, Noel JP, Coates RM, Chappell J. Functional characterization of premnaspirodiene oxygenase, a cytochrome P450 catalyzing regio- and stereo-specific hydroxylations of diverse sesquiterpene substrates. J Biol Chem. 2007;282(43):31744.

56. Croteau R, Ketchum REB, Long RM, Kaspera R, Wildung MR. Taxol biosynthesis and molecular genetics. Phytochem Rev. 2006;5(1):75-97.

57. Søren B, Fred B, Gerard B, Björn H, René H, Suzanne P, Danièle WR. Cytochromes P450. The Arabidopsis Book. 2011;9:e0144. Published By: The American Society of Plant Biologists. https://doi.org/10.1199/tab.0144. http:// www.bioone.org/doi/full/10.1199/tab.0144. Accessed 6 Oct 2011.

58. Hamberger B, Bak S. Plant P450s as versatile drivers for evolution of speciesspecific chemical diversity. Philos Trans R Soc Lond B Biol Sci. 2013; 368(1612):20120426.

59. Ralston L, Kwon ST, Schoenbeck M, Ralston J, Schenk DJ, Coates RM, Chappell J. Cloning, heterologous expression, and functional characterization of 5-epi-aristolochene-1,3-dihydroxylase from tobacco (Nicotiana tabacum). Arch Biochem Biophys. 2001;393(2):222.

60. Wang Y, Hamers RJ. Molecular cloning and functional identification of $(+)-\delta$ cadinene-8-hydroxylase, a cytochrome P450 mono-oxygenase (CYP706B1) of cotton sesquiterpene biosynthesis. Plant J. 2001;28(1):95.

61. Fengnian $Y$, Sho O, Hisashi H, Kazuhisa Y, Norihiko M, Ryutaro U. Zingiber zerumbet CYP71BA1 catalyzes the conversion of a-humulene to 8-hydroxya-humulene in zerumbone biosynthesis. Cell Mol Life Sci. 2011;68(6):1033.

62. Ikezawa N, Göpfert JC, Nguyen DT, Kim SU, O'Maille PE, Spring O, Ro DK. Lettuce Costunolide synthase (CYP71BL2) and its homolog (CYP71BL1) from sunflower catalyze distinct Regio- and Stereoselective Hydroxylations in Sesquiterpene lactone metabolism. J Biol Chem. 2011;286(24):21601.

63. Q L, M M, K C, M G, T C, FW V, et al. Reconstitution of the costunolide biosynthetic pathway in yeast and Nicotiana benthamiana. PLoS One. 2011; 6(8):e23255.

64. Nguyen TD, Macnevin G, Ro DK. De novo synthesis of high-value plant sesquiterpenoids in yeast. Methods Enzymol. 2012;517(517):261-78.

65. Bontpart T, Cheynier V, Ageorges A, Terrier N. BAHD or SCPL acyltransferase? What a dilemma for acylation in the world of plant phenolic compounds. New Phytol. 2015;208(3):695-707.

66. D Auria JC. Acyltransferases in plants: a good time to be BAHD. Curr Opin Plant Biol. 2006;9(3):331-40

67. Sarker LS, Mahmoud SS. Cloning and functional characterization of two monoterpene acetyltransferases from glandular trichomes of $\mathrm{L} . \mathrm{X}$ intermedia. Planta. 2015;242(3):1-11.

68. Hampel D, Mau CJ, Croteau RB. Taxol biosynthesis: identification and characterization of two acetyl CoA:taxoid-O-acetyl transferases that divert pathway flux away from Taxol production. Arch Biochem Biophys. 2009; 487(2):91.

69. Walker K, Croteau R. Taxol biosynthesis: molecular cloning of a benzoyl-CoA: taxane 2alpha-O-benzoyltransferase cDNA from taxus and functional expression in Escherichia coli. P Natl Acad Sci Usa. 2000;97(25):13591-6.

Ready to submit your research? Choose BMC and benefit from:

- fast, convenient online submission

- thorough peer review by experienced researchers in your field

- rapid publication on acceptance

- support for research data, including large and complex data types

- gold Open Access which fosters wider collaboration and increased citations

- maximum visibility for your research: over $100 \mathrm{M}$ website views per year

At $\mathrm{BMC}$, research is always in progress.

Learn more biomedcentral.com/submissions 